\title{
PRODUÇÃO DE CONCENTRADOS DE FRUTOSE POR INULINASES DE Penicillium janczewskii E ATIVIDADE SOBRE O NÍVEL DE GLICOSE PLASMÁtica EM RATOS DIABÉticos ${ }^{1}$
}

\author{
Rosemeire A. Bom PESSONI², Pedro M. O. OLMEDO², Ana C. CLEMENTE FILHA ${ }^{3}$,
}

\author{
Rita C. L. FIGUEIREDO-RIBEIRO ${ }^{3, *}$
}

\section{RESUMO}

A frutose é utilizada atualmente como adoçante para diabéticos, sendo produzida comercialmente por hidrólise do amido, sob um processo de alto custo que envolve três etapas enzimáticas usando $\alpha$-amilase, amiloglicosidase e glicose isomerase. Uma alternativa para a produção de concentrados de frutose é a hidrólise enzimática da inulina, polímero de frutose encontrado em Asteráceas, incluindo espécies nativas do cerrado. Nesse caso, através de uma única etapa enzimática obtêm-se concentrados com até 95\% de frutose. Embora baixos niveis desse açúcar possam ser metabolizados na ausência de insulina, seu efeito sobre a redução do nível de glicose plasmática ainda não está completamente esclarecido. No presente trabalho foi avaliada a ação da frutose produzida por hidrólise da inulina de Vernonia herbacea (Asteraceae) por inulinases de Penicillium janczewskii no nível de glicose plasmática de ratos diabéticos. Dentre os animais diabéticos tratados não foi verificada mortalidade, havendo redução de $46 \%$ em média ( $\mathrm{p}<1 \%$ no teste de Tukey) dos níveis de glicose plasmática quando comparados aos controles. A considerável produção de inulina em $V$. herbacea, a alta atividade inulinásica de $P$. janczewskii e a ausência de mortalidade dos animais tratados demonstram que a frutose obtida por essa via pode ser uma alternativa viável para a produção comercial desse açúcar.

Palavras-chave: fungos filamentosos; inulina; enzimas; hidrolases; microrganismos; hipoglicemiante.

\section{SUMMARY}

FRUCTOSE SYRUPS PRODUCED BY INULINASES FROM PenicilliumjanczewskiiAND ACTIVITY ON PLASMA GLUCOSE LEVEL IN DIABETIC RATS. Fructose has been used as sweetener by patients with diabetes. This sugar is usually produced from starch by a high-cost enzymatic process, which envolves the utilization of $\alpha$-amylase, amyloglucosidase and glucose isomerase. Fructose can be alternatively produced by the enzymatic hydrolysis of inulin, a polymer of fructose stored as reserve in a number of Asteraceae species. Using only one enzymatic step, inulin can be converted into syrups containing up to 95\% fructose. In the present work, fructose syrup was produced from inulin of Vernonia herbacea by hydrolysis with extracellular inulinases from Penicillium janczewskii and evaluated with respect to the effect on plasma glucose level in diabetic rats. Reduction of ca. $46 \%$ ( $<<1 \%$ Tukey test) of glucose levels in the plasma and no mortality were observed when rats were treated with hydrolysate of inulin. The high amounts of inulin stored by $V$. herbacea, the high activity of inulinase from P. janczewskii and the absence of mortality in these assays indicate that fructose produced in this way can be a suitable alternative for the commercial production of fructose syrup.

Keywords: filamentous fungus; inulin; enzyme; microorganism; hydrolases; anti-hyperglycemic.

\section{1 - INTRODUÇÃO}

A frutose é um monossacarídeo com ampla distribuição na natureza e possui um poder adoçante cerca de $70 \%$ superior ao da sacarose [7]. Sua utilização na indústria de alimentos e bebidas vem crescendo de forma acentuada, pois esse açúcar não apresenta problemas de cristalização como a sacarose [12]. Além disso, a frutose é tolerada por pacientes diabéticos e está relacionada com o aumento da absorção de ferro em crianças [24]. Dessa forma, a frutose vem sendo recomendada como adoçante alternativo para diabéticos, uma vez que promove diminuição do nível de glicose plasmática [1] e, diferentemente da glicose, pode ser metabolizada independentemente da insulina [2].

Comercialmente, a frutose é produzida a partir da isomerização da glicose, que por sua vez é obtida por

\footnotetext{
Recebido para publicação em 12/12/2002. Aceito para publicação em 31/05/2004 (001028).

2. Universidade Metodista de São Paulo, Caixa Postal 5002, CEP: 09735-460, São Bernardo do Campo-SP, Brasil. E-mail: pessoni@ metodista.br

3. Instituto de Botânica, Caixa Postal 4005, CEP: 01061-970, São PauloSP, Brasil. E-mail: ritarib@usp.br

* A quem a correspondência deve ser enviada.
}

hidrólise multi-enzimática do amido [13]. Uma alternativa para esse processo é a hidrólise da inulina, polímero de frutose encontrado como carboidrato de reserva em várias plantas da família asterácea, como Cichorium intybus e Helianthus tuberosus [17, 27] e em espécies nativas do cerrado brasileiro, como Vernonia herbacea e Viguiera discolor, sendo que nestas últimas cerca de $80 \%$ da massa seca de seus órgãos subterrâneos são constituídos por inulina [11, 14]. Para a obtenção de frações com características biológicas similares, derivadas do polissacarídeo inulina, é importante a utilização de um método que permita a diminuição do grau de polimerização do polissacarídeo sem, contudo, modificar suas características estruturais, para que a ação biológica não seja afetada. A utilização de enzimas específicas é a mais indicada. Nesse particular, a inulina pode ser despolimerizada pela atividade de exoinulinases (EC 3.2.1.80) ou por endoinulinases (EC 3.2.1.7) secretadas por fungos, leveduras ou bactérias, sendo que a hidrólise completa da inulina por exoinulinases produz um concentrado de frutose com cerca de 95\% de pureza, em uma única etapa enzimática [16].

Enzimas que degradam inulina foram inicialmente purificadas a partir de plantas, mas as quantidades obtidas eram insuficientes para serem exploradas comercialmente. Por esse motivo, inulinases produzidas por microrganismos, que podem ser induzidas pelas 
condições de cultivo têm recebido especial atenção na última década $[18,23]$.

Penicillium janczewskii Zaleski é um fungo filamentoso, originalmente isolado da rizosfera de Vernonia herbacea, e que cresce rapidamente em meio contendo inulina como fonte de carbono, sendo bastante eficiente na produção de inulinases [25].

No presente trabalho foi avaliada a ação de concentrados de frutose produzidos a partir da hidrólise da inulina de Vernonia herbacea por inulinases extracelulares de Penicillium janczewskii no nível de glicose plasmática em ratos Sprague Dawley.

\section{2 - MATERIAL E MÉTODOS}

\section{1 - Material biológico}

O fungo Penicillium janczewskii Zaleski (URM - 3511), utilizado neste trabalho, foi isolado da rizosfera de plantas herbáceas da família Asteraceae, a partir de suspensões de solos coletados no cerrado da Reserva Biológica e Estação Experimental de Moji-Guaçu - SP e em regiões de cerrado nos municípios de Itirapina - SP e de São Carlos - SP [6]. Culturas do fungo foram mantidas em meio de cultura sólido (Batata-Dextrose-Agar) e em óleo mineral na Seção de Fisiologia e Bioquímica de Plantas do Instituto de Botânica de São Paulo.

\section{2 - Obtenção de inulina de Vernonia herbacea}

Amostras de rizóforos de Vernonia herbacea (Vell.) Rusby (Asteraceae) foram coletadas na Reserva Biológica e Estação Experimental de Moji-Guaçu. As amostras foram fragmentadas e imediatamente submersas em etanol $80 \%$ por $3 \mathrm{~min}$, homogeneizadas em liquidificador por 5min e filtradas à vácuo. O resíduo foi extraído duas vezes com água destilada, em banho-maria a $60^{\circ} \mathrm{C}$, por $30 \mathrm{~min}$. Os filtrados foram reunidos e concentrados em evaporador rotatório para redução do volume; o material foi tratado com 3 volumes de etanol, durante uma noite a $5^{\circ} \mathrm{C}$, para precipitação dos polissacarídeos. Após o pernoite, foi submetido à centrifugação a $8000 \mathrm{~g}$, por $15 \mathrm{~min}$, a $5^{\circ} \mathrm{C}$ [4]. O sobrenadante foi descartado e o precipitado foi seco por liofilização e utilizado como substrato para o cultivo do fungo e nos ensaios para obtenção dos concentrados de frutose.

\section{3 - Obtenção de inulinases de $P$. janczewskii e produção de concentrados de frutose}

A obtenção das enzimas extracelulares de $P$. janczewskii foi baseada nos trabalhos de KOCHHAR, GUPTA \& KAUR [18] e PESSONI, FIGUEIREDO-RIBEIRO \& BRAGA [25]. Após o cultivo por 12 dias em meio líquido contendo inulina de $V$. herbacea como fonte de carbono, os meios de cultura foram separados da massa micelial por filtração em papel de filtro, seguido por filtração em "millipore" em filtros de $0,45 \mu \mathrm{m}$. O resíduo foi descartado e o filtrado precipitado com sulfato de amônio (30-80\% de saturação) e centrifugado por $20 \mathrm{~min}$ a $10.000 \mathrm{~g}$ a $5^{\circ} \mathrm{C}$. O precipitado foi retomado em pequeno volume de tampão Tris- $\mathrm{HCl} 20 \mathrm{mM}$ e dessalinizado em coluna de Bio-gel P6DG, previamente preparada no mesmo tampão. Após dessalinização, o extrato foi aplicado em coluna de troca iônica em sistema FPLC utilizando uma coluna Mono $Q$ (HR 5/5 - Pharmacia), equilibrada com tampão Tris- $\mathrm{HCl} 20 \mathrm{mM}$ ( $\mathrm{pH} 7,8$ ). As enzimas foram eluídas através de gradiente linear de cloreto de sódio de $0-0,5 \mathrm{M}$, com fluxo de $1 \mathrm{~mL} / \mathrm{min}$, sendo as frações reunidas e dessalinizadas em coluna de Bio-gel P6DG. Esta solução foi utilizada como fonte de enzimas nos ensaios para a obtenção dos concentrados de frutose.

A inulina de $V$. herbacea (2g) foi hidrolisada pela solução enzimática $(3 \mathrm{U} / \mathrm{mL})$ durante 26 horas a $55^{\circ} \mathrm{C}$ em pH 5,0, conforme previamente determinado PESSONI, FIGUEIREDO-RIBEIRO \& BRAGA [25]. A atividade enzimática da solução foi quantificada pelo poder redutor liberado, através de determinação dos açúcares redutores pelo método de SOMOGYI [29], utilizando frutose como padrão. A mistura de incubação foi concentrada em evaporador rotatório até secagem relativa e ressuspendida em água destilada, sendo considerada como o concentrado de frutose, que foi utilizado para avaliação da atividade biológica.

Alíquotas da solução enzimática foram dessalinizadas e filtradas em membranas específicas para HPLC,

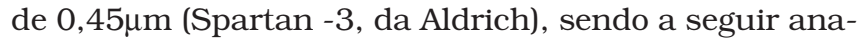
lisadas em sistema de cromatografia aniônica de alto desempenho, acoplado a detector amperométrico pulsado (HPAEC/PAD), Mod. DX-300, da Dionex, em coluna Carbo Pac PA-1. Como eluentes foram utilizados gradientes de $\mathrm{NaOH} 150 \mathrm{mM}$ (eluente A) e de acetato de sódio 500mM em $\mathrm{NaOH} 150 \mathrm{mM}$ (eluente B), conforme estabelecido por SHIOMI [28]. As amostras foram preparadas na concentração de $400 \mathrm{mg}$ de equivalente de frutose por mililitro de água deionizada e quantificadas pelo método de antrona modificado por JERMYN [15]. Este material foi comparado com concentrado de frutose comercial (Extravita Lab.Nutricional S/A).

\section{4 - Indução do diabetes, administração do con- centrado de frutose e quantificação de glicose plas- mática}

A metodologia utilizada para a indução de diabetes e avaliação do nível de glicose plasmática foi baseada em PEPATO et al. [21].

Foram utilizados 18 ratos machos Sprague Dawley com cerca de $180 \mathrm{~g}$ e mantidos em condição padrão de biotério, temperatura de $25^{\circ} \mathrm{C}, 55 \%$ de umidade relativa e ciclo $12 \mathrm{~h}$ luz-escuro, com acesso livre à água e alimento.

O diabetes foi induzido com estreptozotocina (Sigma) em concentração de $40 \mathrm{mg} / \mathrm{kg}$ de massa corporal, sendo injetada na veia dorsal peniana dos animais mantidos em jejum por 14-16 horas. Após três dias os animais foram aleatoriamente separados em dois grupos. O grupo tratado (9 animais) recebeu 100mg de concentrado de frutose $/ \mathrm{kg}$ de massa corporal e o grupo controle recebeu água destilada. A administração do concentrado de frutose ou de água foi realizada via intraperitoneal, em dose única. 
A quantificação da glicose no sangue foi realizada nos tempos 3,5 e 7 horas após a administração do concentrado de frutose. O sangue foi coletado da veia caudal, com anticoagulante e a seguir centrifugado por $5 \mathrm{~min}$, a 3.000rpm. O plasma foi utilizado para quantificação de glicose pelo método enzimático da glicose oxidase (Labtest Diagnóstica).

A análise estatística foi realizada pelo programa SANEST [32].

\section{5 - Testes de toxicidade}

A inulina de $V$. herbacea também foi submetida a testes farmacológicos preliminares no Departamento de Psicobiologia da Universidade Federal de São Paulo (UNIFESP) utilizando camundongos machos albinos de linhagem Suiça com 3 meses de idade, não diabéticos. Nesse teste foram observados alguns parâmetros, conforme MATTEI et al. [19], como a presença ou ausência de micção, defecação, atividade motora, ptose palpebral, tônus muscular, tremores e mortalidade após $48 \mathrm{~h}, 72 \mathrm{~h}$ e 7 dias.

\section{3 - RESULTADOS E DISCUSSÃO}

Segundo PESSONI, FIGUEIREDO-RIBEIRO \& BRAGA [25], a inulina de Vernonia herbacea pode ser um substrato ideal para o cultivo e indução de inulinases de Penicillium janczewskii, visto que estimula alta atividade inulinásica quando comparada com outras fontes de carbono.

A inulinase semi-purificada de $P$. janczewskii, utilizada para a obtenção do concentrado de frutose, continha $26 \mathrm{U}$ de atividade específica após cromatografia em coluna de troca aniônica (Tabela 1). KOCHHAR, GUPTA \& KAUR [18], estudando a produção de inulinases extracelulares por diversos fungos, selecionaram Aspergillus candidus como o microrganismo ideal para utilização industrial, visando à produção de concentrados de frutose. A atividade inulinásica obtida para $P$. janczewskii cultivado durante 12 dias em meio líquido foi comparada aos resultados obtidos por aqueles autores, cujos dados estão mostrados na Tabela 1.

TABELA 1. Inulinases extracelulares produzidas por diferentes fungos, durante 12 dias de cultivo em meio líquido.

\begin{tabular}{lccc}
\hline Fungos & $\begin{array}{c}\mathrm{U} / \mathrm{L} \\
\text { Meio de cultura }\end{array}$ & $\begin{array}{c}\mathrm{U} / \mathrm{mg} \text { protel̀na } \\
\text { Extrato bruto }\end{array}$ & $\begin{array}{c}\text { U/mg protelna } \\
\text { Extrato semi- } \\
\text { purificado }\end{array}$ \\
\hline Penicillium janczewskii & 1380 & 15 & 26 \\
Aspergillus candidus & 9046 & 2,1 & 21,2 \\
Aspergillus chevalieri & 31 & - & - \\
Aspergillus oryzae & 95 & - & - \\
Aspergillus terreus & 98 & - & - \\
Fusarium moniliforme & 109 & - & - \\
Mucor rouxii & 63 & - & - \\
Neurospora crassa & 11 & - & - \\
Penicillum funiculosum & 3176 & - & - \\
Rhizopus oryzae & 69 & - & - \\
\hline
\end{tabular}

* Penicillium janczewskii: valores obtidos no presente trabalho. Os demais fungos e respectivos valores são oriundos da literatura [18]
Como pode ser observado na Tabela 1, o P.janczewskii apresentou alta atividade inulinásica quando comparado aos demais fungos estudados por KOCHHAR, GUPTA \& KAUR [18], exceto A. candidus e $P$. funiculosum. Com relação à atividade específica nos extratos brutos, o valor encontrado para P. janczewskii foi superior ao obtido para A. candidus. Após purificação parcial, em coluna de troca aniônica, o valor obtido para $P$. janczewskii foi $26 \mathrm{U} / \mathrm{g}$ proteína sendo semelhante ao observado para A. candidus, cuja atividade foi $21 \mathrm{U} / \mathrm{mg}$ proteína, utilizando os mesmos passos de purificação.

CRUZ et al. [7] estudando 350 linhagens de Aspergillus niger selecionaram A. niger-245, cuja atividade inulinásica foi $2,92 \mathrm{U} / \mathrm{mL}$, após 60 horas de cultivo, sendo considerado por eles como um microrganismo interessante para utilização industrial. O meio de cultura utilizado pelos autores era enriquecido com peptona como fonte de nitrogênio. PESSOA JR. \& VITOLO [24] também consideraram Kluyveromyces marxianus um microrganismo promissor para produção de inulinases em escala industrial. Esses autores enriqueceram o meio de cultura com diferentes fontes de nitrogênio (peptona ou extrato de levedura) e, conseguiram aumentar a produção de inulinases, obtendo uma enzima com atividade entre $12-26 \mathrm{U} / \mathrm{mL}$ de meio de cultura. O enriquecimento do meio de cultura de $P$. janczewskii com diferentes fontes de nitrogênio, bem como a utilização de alta concentração de inulina poderiam também contribuir para o aumento da produção de inulinases por este fungo.

Os produtos de hidrólise da inulina pelas inulinases extracelulares de $P$. janczewskii após 26 horas de incubação e análise por HPAEC/PAD estão mostrados na Figura $1 b$. O material foi comparado com o concentrado de frutose comercial (Figura 1c), sendo notável a semelhança dos perfis cromatográficos dos dois concentrados. É importante destacar que os concentrados de frutose obtidos a partir da hidrólise enzimática da inulina de $V$. herbacea continham traços de glicose, resultantes da hidrólise da sacarose, que faz parte da molécula da inulina.

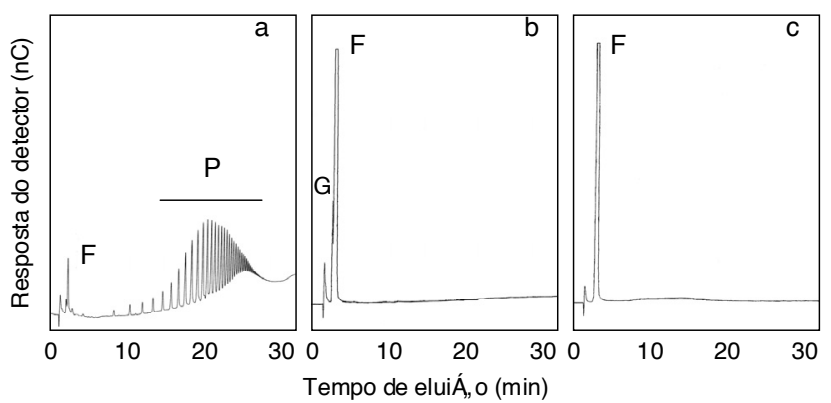

O perfil cromatográfico representa o sinal do detector amperométrico pulsado (PAD) em nano Coulombs (nC). a - tempo zero; b - 26 horas de incubação; c - concentr

FIGURA 1. Cromatografia aniônica de alta resolução (HPAEC/ PAD) dos produtos de incubação do extrato enzimático de $P$. janczewskii com inulina de $V$. herbacea.

As características das inulinases de P. janczewskii, como alta atividade em $\mathrm{pH}$ ácido, termoestabilidade e 
alta afinidade por inulina de $V$. herbacea [25], conferem vantagens para uma utilização industrial do $P$. janczewskii visando à produção de concentrados de frutose. Segundo KATO et al. [16], altas temperaturas, além de inibir a contaminação bacteriana, auxiliam a solubilização de inulina e o pH ácido impede a formação de compostos coloridos.

Inulina é fisiologicamente inerte e não tóxica em doses fisiológicas e é considerada como substância ideal para medidas de filtração glomerular, tanto em modelos animais, como no homem [3]). DIAS-TAGLIACOZZO, DIETRICH \& MELLO-AIRES [8] submeteram a inulina de $V$. herbacea a um teste de filtração glomerular e mostraram que este polissacarídeo é um substrato alternativo à inulina comercial (Sigma) utilizada para essa finalidade. Testes farmacológicos preliminares realizados no Departamento de Psicobiologia da UNIFESP indicaram que a inulina de $V$. herbacea, nas doses de 10 e $100 \mathrm{mg} / \mathrm{kg}$, não apresentam diferenças quanto à micção, defecação, atividade motora, ptose palpebral, tônus muscular, tremores e mortalidade dos ratos tratados em relação ao grupo controle (R. Mattei, Comunicação pessoal). No presente trabalho também não ocorreram alterações fisiológicas aparentes nos ratos que receberam concentrado de frutose produzido a partir da hidrólise desse polissacarídeo.

Com base nos resultados laboratoriais e face aos dados de boa produtividade da espécie em campo [5], $V$. herbacea representa um recurso natural do cerrado para fins de exploração econômica e utilização como substrato alternativo e de baixo custo para a produção de concentrados de frutose. Atualmente esses concentrados são produzidos a partir da hidrólise enzimática do amido de milho, em três etapas enzimáticas envolvendo $\alpha$-amilase, amiloglicosidase e glicose isomerase, produzindo um concentrado com cerca de $45 \%$ de frutose [20], enquanto a hidrólise da inulina pode produzir um concentrado com cerca de $95 \%$ de frutose. Sabe-se que a inulina poderia ser hidrolisada quimicamente para a obtenção desses xaropes de frutose, porém resulta na formação de cerca de $5 \%$ de anidrido di-frutose, que não tem poder adoçante, e que não pode ser hidrolisado até frutose, devendo ser retirado da preparação [18].

Embora baixos niveis de frutose possam ser metabolizados na ausência de insulina, seus efeitos sobre a redução dos níveis de açúcar no sangue são discutiveis. A glicose estimula a secreção de insulina, mas outros hormônios e neurotransmissores podem agir sobre as células $\beta$ das ilhotas pancreáticas e modular o processo de secreção [22]. A glicose é fosforilada a glicose-6-fosfato durante a primeira reação da via glicolítica, que é catalisada pela enzima glucoquinase (GK) do fígado. A expressão do gene da GK no fígado é positivamente estimulada pela insulina [9]. Entretanto, VAN SCHAFTINGEN \& VANDERCAMMEN [31] verificaram que baixas concentrações de frutose estimulam a atividade de GK de hepatócitos, em ausência de insulina; o efeito é mediado pela frutose-1-fosfato, que antagoniza a inibição da glucoquinase causada por uma proteína regulatória específica [26].
A dose de $40 \mathrm{mg} / \mathrm{kg}$ de estreptozotocina mostrou-se adequada para a indução do diabetes em ratos Sprague Dawley com $180 \mathrm{~g}$ de peso, visto que três dias após a administração da droga todos os animais atingiram níveis de glicose sangüinea entre 200 - 250mg/dL. Os animais diabéticos tratados com frutose demonstraramse hígidos e em todos os tempos analisados houve redução significativa ( $\mathrm{p}<1 \%$ no teste de Tukey) dos níveis de glicemia em relação aos controles (Figura 2).

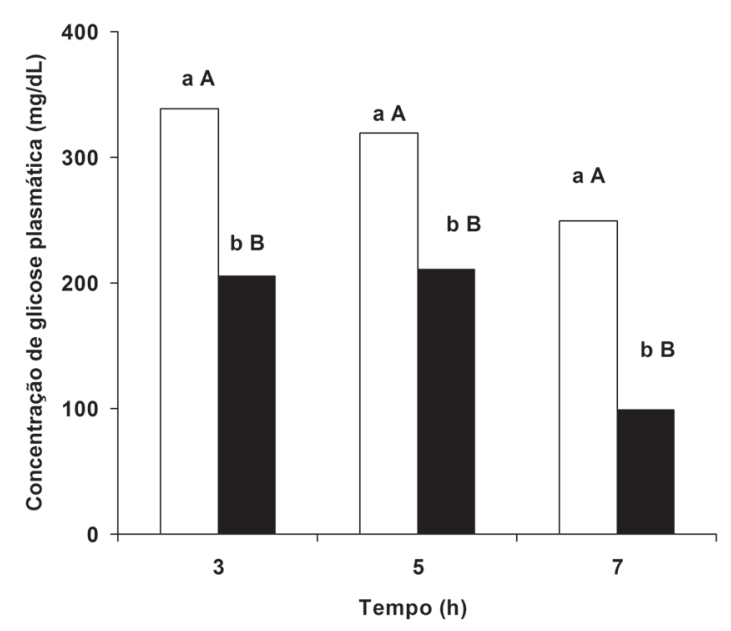

As letras minúsculas compararam controle $(\square)$ e tratado $(\square)$ em cada tempo e as letras maiúsculas comparam controle ou tratado nos diferentes tempos de análise. (Teste de Tukey , $\mathrm{p}<1 \%, \mathrm{n}=9$ ).

FIGURA 2. Efeito do concentrado de frutose, obtido por hidrólise de inulina de $V$. herbacea por inulinases de $P$. janczewskii, no nível de glicose plasmática de ratos machos Sprague Dawley diabéticos.

Os resultados obtidos concordam com dados da literatura que mostram que frutose em baixas concentrações pode reduzir níveis de glicose do sangue [10]. Recentemente foi verificado que animais submetidos a dietas com alto teor de frutose apresentaram aumento do nível de triglicérides plasmáticos [30], alertando para o fato de que a utilização de concentrados de frutose em dietas alimentares de diabéticos precisa ser dosada e estar sob vigilância.

\section{4 - CONCLUSÕES}

- O concentrado de frutose produzido pela hidrólise da inulina de $V$. herbacea foi similar ao produto comercial e demonstrou diminuir o nível de glicose plasmática em todos os tempos analisados.

- O considerável acúmulo de inulina em Vernonia herbacea (até $80 \%$ da massa seca), a alta atividade inulinásica de Penicillium janczewskii, produzindo concentrados de frutose de alta pureza e a ausência de alterações fisiológicas dos animais tratados demonstram que a frutose obtida pela hidrólise enzimática da inulina de $V$. herbacea é uma alternativa viável e que deve ser considerada para a produção comercial de frutose. a partir do cultivo desta espécie do cerrado. 


\section{5 - REFERÊNCIAS BIBLIOGRÁFICAS}

[1] AKGÜN, S.; ERTEL, N.H. The effects of sucrose, fructose, and high-fructose corn syrup meals on plasma glucose and insulin in non-insulin-dependent diabetic subjects. Diabetes Care, v. 8, p. 279-283, 1985.

[2] BLAKELY, S.T.; MISLO, B.L.; BASI, N.S.E.; PAINTER, R.H. Dietary fructose alters the inulin-like effects of dietary vanadate in adipocytes from rats. Nutr. Res, v. 15, p. 25-35, 1995.

[3] BRENNER, B.M.; DWORKIN, L.D.; ICHIKAWA, I. Glomerular ultrafiltration. In: BRENNER, M.D.; FLOYD, C.; RECTOR, M.D.; editors. Philadelphia, USA: The Kidney, WB Saunders. p. 124-144, 1986.

[4] CARVAlHO, M.A.M.; DIETRICH, S.M.C. Variation in fructan content in the underground organs of Vernonia herbacea (Vell.) Rusby at different phenological phases. New Phytol, v. 123, p. 735-740, 1993.

[5] CARVALHO, M.A.M.; PINTO, M.M.; FIGUEIREDO-RIBEIRO, R.C.L. Inulin production by Vernonia herbacea as influenced by mineral fertilization and time of harvest. Rev. Bras. Bot., v. 21, p. 275-280, 1998.

[6] CORDEIRO-NETO, F.; PESSONI, R.A.B.; FIGUEIREDORIBEIRO, R.C.L. Fungos produtores de inulinases isolados da rizosfera de asteráceas herbáceas do cerrado. Rev. Bras. Ci. Solo, v. 21, p. 149-153, 1997.

[7] CRUZ, V.D.; BELOTE, J.G.; BELLINE, M.Z.; CRUZ, R. Production and action pattern of inulinase from Aspergillus niger-245: Hydrolysis of inulin from several sources. Rev. Microbiol., v. 29, p. 301-306, 1998.

[8] DIAS-TAGLIACOZZO, G.M.; DIETRICH, S.M.C.; MELLOAIRES, M. Measurement of glomerular filtration rate using inulin prepared from Vernonia herbacea, a Brazilian native species. Braz. J. Med. Biol. Res., v. 29, p. 13931396, 1996.

[9] DOIRON, B.; CUIF, M.H.; KAHN, A.; DIAZ-GUERRA, M.J.M. Respective roles of glucose, fructose, and insulin in the regulation of the liver-specific pyruvate kinase gene promoter. J. Biol. Chem., v. 269, p. 10213-10216, 1994.

[10] FRAIGE-FILHO, R.; BLANDY, A.A.; FERNANDES, C.; LUZ, L.T.; MARINI, A.M.; SALAROLI, G.R. Avaliação da utilização da frutose na dieta de pacientes diabéticos. Arq. Med. ABC, v. 1-2, p. 19-21, 1996.

[11] FIGUEIREDO-RIBEIRO, R.C.L.; DIETRICH, S.M.C.; CHU, E.P.; CARVALHO, M.A.M.; VIEIRA, C.C.J.; GRAZIANO, T.T. Reserve carbohydrate in underground organs of native Brazilian plants. Rev. Bras. Bot., v. 9, p. 159$166,1986$.

[12] GE, Y.; ZHOU, H.; KONG, W.; TONG, Y.; WANG, S.; LI, W. Immobilization of glucose isomerase and its application in continuous production of high fructose syrup. Appl. Biochem. Biotech., v. 69, p. 203-215, 1998.

[13] GODFREY, T.; WEST, S. Industrial Enzimology. New York: Stockton Press. 1996, 609p.

[14] ITAYA, N.M.; FIGUEIREDO-RIBEIRO, R.C.L.; BUCKERIDGE, M.S. Synthesis of fructans by fructosyltransferase from the tuberous roots of Viguiera discolor (Asteraceae). Braz. J. Med. Biol. Res., v. 32, p. 435-442, 1999.

[15] JERMYN, M.A. A new method for the determination of ketohexoses in presence of aldohexoses. Nature, v. 177, p. 38-39, 1956.

[16] KATO, K.; ARAKI, T.; KITAMURA, T.; MORITA, N.; MOORI, M.; SUZUKI, Y. Purification and properties of a thermostable inulinase ( $\beta$-D-fructan fructohydrolase) from Bacillus stearothermophilus KP 1289. Starch, v. 51, p. 253-258, 1999.
[17] KAUR, N.; GUPTA, A.K. Applications of inulin and oligofructose in health and nutrition. J. Biosci., v. 27, p. 703-714, 2002.

[18] KOCHHAR, A.; GUPTA, A.K.; KAUR, N. Purification and immobilisation of inulinase from Aspergillus candidus for producing fructose. J. Sci. Food Agric., v. 79, p. 549-554, 1999.

[19] MATTEI, R.; DIAS, R.F.; ESPÍNOLA, E.B.; CARLINI, E.L.A.; BARROS, S.B.M. Guaraná (Paullinia cupana): behavioral and toxic effects on laboratory animals and in vitro antioxidant activity. J Ethnopharmacol (Irlanda), v. 60, p. 111-116, 1998.

[20] Pandey A, Soccol CR, Selvakumar P, Soccol VT, Krieger N, Fontana JD. 1999. Recent developments in microbial inulinases. Appl. Biochem. Biotechn., v. 81, p. 3552.

[21] PEPATO, M.T.; OlIVEIRA, J.R.; KeTtELHUT, E.C.; MIGLIORINI, R.H. Assessment of the antidiabetic activity of Myrcia uniflora extracts in streptozotocin diabetic rats. Diabetes Res., v. 22, p. 49-57, 1993.

[22] PERSAUD, S.J.; ASARE-ANANE, H.; JONES, P.M. Insulin receptor activation inhibits insulin secretion from human islets of Langerhans. FEBS Lett, v. 510, p. 225-228, 2002.

[23] PESSOA-JR, A.; VITOLO, M. Recovery of inulinase using BDBAC reversed micelles. Proc. Biochem., v. 33, p. 291297, 1998

[24] PESSOA-JR, A.; VITOLO, M. Inulinase from Kluyveromyces marxianus: culture medium composition and enzyme extraction. Braz. J. Chem. Eng., v. 16, p. 237-245, 1999.

[25] PESSONI, R.A.B.; FIGUEIREDO-RIBEIRO, R.C.L.; BRAGA, M.R. Extracellular inulinases from Penicillium janczewskii, a fungus isolated from the rhizosphere of Vernonia herbacea (Asteraceae). J. Appl. Microbiol., v. 86, p. 1024-1032, 1999.

[26] PHILliPS, J.W.; HENLY, D.C.; BERRY, M.N. Longterm maintenance of low concentrations of fructose form the study of hepatic glucose phosphorylation. Biochem. J., v. 337, p. 4977-501, 1999.

[27] SELVAKUMAR, P.; PANDEY, A. Solid state fermentation for the synthesis of inulinase from Staphylococcus sp. and Kluyveromyces marxianus. Proc. Biochem., v. 34, p. 851-855, 1999.

[28] SHIOMI, N. Structure of fructo-polysaccharide (asparagosin) from roots of asparagus (Asparagus officinalis L.). New Phytol., v. 123, p. 263-270, 1993.

[29] SOMOGYI, M. A new reagent for the determination of sugar. J. Biol. Chem., v. 160, p. 61-68, 1945.

[30] UENO, M.; BEZERRA, R.M.N.; SIVAL, M.S.; TAVARES, D.Q.; CARVALHO, C.R.; SAAD, M.J.A. A high-fructose diet induces changes in pp 185 phosphorylation in muscle and liver of rats. Braz. J. Med. Biol. Res., v. 33, p. 1421-1427, 2000.

[31] VAN SCHAFTINGEN, E.; VANDERCAMMEN, A. Stimulation of gluxose phosphorylation by fructose in isolated rat hepatocytes. E. J. Biochem., v. 179, p. 173177, 1989.

[32] ZONTA, E.P.; MACHADO, A.A. SANEST - Sistema de análises estatísticas. Piracicaba, ESALQ/USP, 1982.

\section{6 - AGRADECIMENTOS}

Agradecemos à Profa. Dra. Rita Mattei, do Departamento de Psicobiologia da Universidade Federal de São Paulo (UNIFESP) pelos testes de toxicidade. 